\title{
Recent Developments and Limitations of the SFG Model
}

\author{
D. Sheng
}

\begin{abstract}
The SFG model was proposed in attempt to provide a consistent description of the stress-strain behaviour of unsaturated soils, including compacted soils and soils dried from slurry. It differs from existing models mainly through two aspects: a consistent description of volume change, yield stress and shear strength behaviour of unsaturated soils and a smooth and natural transition between saturated and unsaturated states. The model has attracted significant attention since it was first proposed and has been extended to cover coupled hydro-mechanical behaviour, hysteretic water retention behaviour and density-dependency. This paper presents a summary of the latest developments of the model, as well as the aspects that require further refinements.
\end{abstract}

Keywords: SFG model, unsaturated soils, volume change, shear strength, hydro-mechanical coupling.

\section{Introduction}

The SFG model proposed by Sheng et al. (2008a) attempts to provide a consistent and unified description of volume change, yield stress, shear strength and hydraulic behaviour of unsaturated soils, including compacted soils and soils dried from slurry. It is established in the space of the independent stress variables, namely net stress and matric suction. However, unlike models using similar stress variables, the SFG model provides a smooth and natural transition between saturated and unsaturated states. The model has attracted significant attention since it was proposed in 2008. It has been further extended to cover soil behaviour such as hydro-mechanical interaction, hysteretic water retention, and density dependency. However, this model also has a number of limitations that require further improvements. This paper presents a summary of the latest developments of the SFG model and the areas where the model falls short of real soil behaviour.

\section{The SFG Model}

The essential ingredient of the SFG model is its volume change equation. According to the model, the volume change of a soil can be caused by a change of net stress or a change of suction. For normally consolidated soils under isotropic stress states, the volume change equation takes the following form:

$$
d v=-\lambda_{v p} \frac{d \bar{p}}{\bar{p}+f(s)}-\lambda_{v s}(s) \frac{d s}{\bar{p}+f(s)}
$$

where is the specific volume and $v=1+e, e$ is the void ratio, $\bar{p}$ is the mean net stress and $\bar{p}=p-u_{a}, p$ is the mean stress, $u_{\mathrm{a}}$ is the pore air pressure, $u_{w}$ is the pore water pressure, $s$ is the soil suction and $s=u_{a}-u_{w}, \lambda_{v p}$ is the soil compressibility in terms of stress changes, and $\lambda_{v s}$ is the soil compressibility in terms of suction changes. The function $f(s)$ represents the interaction between stress and suction and was simply set to $s$ in the original SFG model (Sheng et al., 2008a). However, more advanced forms could be used, as suggested by Sheng (2011).

The parameter $\lambda_{v p}$ can be determined from normal compression lines (NCL) for $s=0$. It is the same as the slope $(\lambda)$ of NCL in $e-\ln p$ plots that are commonly used for saturated soils. In its simplest form this parameter can be treated as a constant for one soil, but more realistically it should be a function of suction. The parameter $\lambda_{v s}$ is a function of suction. Its initial value is the same as $\lambda_{v p}$ for suctions below the transition value between saturated and unsaturated states, but it approaches zero as suction increases to infinite. The following simple equation was used in Sheng et al. (2008b):

$$
\lambda_{v s}= \begin{cases}\lambda_{v p} & s<s_{s a} \\ \lambda_{v p} \frac{s_{s a}}{s} & s \geq s_{s a}\end{cases}
$$

where $S_{s a}$ is the transition suction and its definition is slightly different from the air entry value (see Sheng et al., 2008a).

The volume change model defined by Eqs. 1 and 2 is the foundation of the SFG model. Zhou \& Sheng (2009) provided a systematic validation of the volume change equation for compacted and air-dry soils. The yield stress and shear strength criteria in the SFG model are all based on this volumetric model. The yield stress function for a slurry soil can be derived from the volume change equation (assuming that the plastic volumetric strain is only hardening variable) and it also defines the apparent tensile strength of the soil:

Daichao Sheng, Professor, School of Engineering, The University of Newcastle, NSW 2308, Australia, and School of Civil Engineering, Central South University, Changsha, 410072, China. e-mail: daichao.sheng@gmail.com.

Invited Article, no discussion. 
$\bar{p}_{0}= \begin{cases}-s & s<s_{s a} \\ -s_{s a}-s_{s a} \ln \frac{s}{s_{s a}} & s \geq s_{s a}\end{cases}$

The loading-collapse equation for collapsible soils (e.g. compacted soils) can also be derived from the volume change equation:

$\bar{p}_{c}= \begin{cases}\bar{p}_{c n 0}-s & s \leq s_{s a} \\ \bar{p}_{c n 0}\left(\bar{p}_{c 0}+f(s)-s_{s a}-s_{s a} \ln \frac{s}{s_{s a}}\right) & s>s_{s a}\end{cases}$

where $\bar{p}_{c}$ is the yield stress at an arbitrary suction, $\bar{p}_{c n 0}$ is the yield stress at zero suction, $\bar{p}_{c 0}$ is a constant.

Note that there is no 'suction-increase' or 'suctiondecrease' yield surface in the SFG model. The plastic volumetric strain caused by drying a soil to historically high suction is automatically covered in the apparent tensile strength function $\bar{p}_{0}$.

The apparent tensile strength function and the loading-collapse function can be incorporated into existing constitutive models for saturated soils. For example, if the modified Cam clay model is used for saturated soil behaviour, the yield function can be generalised to unsaturated states along the suction axis:

$$
f=q^{2}-M^{2}\left(\bar{p}-\bar{p}_{0}\right)\left(\bar{p}_{c}-\bar{p}\right) \equiv 0
$$

where $f$ is the yield function in the stress space, $q$ is the deviator stress, $M$ is the slope of the critical state line in $q-\bar{p}$ space, and $\bar{p}_{0}$ and $\bar{p}_{c}$ are defined above.

Using the shear strength criterion proposed by Fredlund et al. (1978):

$$
\tau=\left[c^{\prime}+\left(\sigma_{n}-u_{a}\right) \tan \phi^{\prime}\right]+\left[\left(u_{a}-u_{w}\right) \tan \phi^{b}\right]
$$

The friction angle due to suction in the SFG model can be derived from the apparent tensile strength equation and is given by

$$
\tan \phi^{b}= \begin{cases}\tan \phi^{\prime} & s<s_{s a} \\ \tan \phi^{\prime}\left(\frac{s_{s a}}{s}+\frac{s_{s a}}{s} \ln \frac{s}{s_{s a}}\right) & s \geq s_{s a}\end{cases}
$$

In the equations above, $\tau$ is the shear strength, $\sigma_{n}$ is the normal stress on the failure plane, $\phi$ ' is the effective friction angle of the soil, and $\phi^{b}$ is the frictional angle due to suction. Equation 7 can be used to predict the change of the shear strength against suction.

In the original SFG model, hydro-mechanical coupling was only considered from the aspect that suction affects the stress-strain behaviour. This was extended to consider the effect of stress on the water retention behaviour by Sheng \& Zhou (2011) by incorporating the following constitutive equation:

$$
d S_{r}=E d s+\frac{S_{r}}{n}\left(1-S_{r}\right)^{m} \frac{\lambda_{v p}}{\bar{p}+f(s)} d \bar{p}
$$

where $S_{r}$ is the degree of saturation, $n$ is the porosity, and $m$ is a fitting parameter. Function $E$ in Eq. 8 refers to the gradient of the soil-water characteristic curve (SWCC, $d S_{r}=E d s$ ) and may also be multi-valued in a piece-wise equation, dependent on the suction path. Because Eq. 8 is in an incremental form, integration of the equation requires one specific SWCC that corresponds to a reference initial void ratio. In other words, the conventional SWCC equation is only used for the reference initial void ratio and the new SWCC for a new initial void ratio is obtained by integration of Eq. 8. Sheng \& Zhou (2011) gives the details on how to use an existing SWCC equation for different initial void ratios.

We note that replacing Eq. 1 into Eq. 8 leads to:

$d S_{r}=\left(E-\frac{\lambda_{v s}}{\bar{p}+f(s)} \frac{S_{r}}{n}\left(1-S_{r}\right)^{m}\right) d s-\frac{S_{r}}{e}\left(1-S_{r}\right)^{m} d e$

The above equation clearly indicates that the $S_{r}-s$ relationship for constant void ratio $(\mathrm{d} e=0)$ is not the same as the SWCC equation $\left(d S_{r}=E d s\right)$. Equations 8 and 9 are equivalent and interchangeable.

Equations 1-9 are the main equations of the SFG model. These equations sufficiently define the volume change, yield stress, shear strength and coupled hydromechanical behaviour of unsaturated soils. In the following, we will first discuss what the SFG model can do and then outline where it falls short.

\section{Predictions of the SFG Model}

The main advantage of the SFG model is that it is based on a simple volume change equation and is able to provide a consistent framework for volume change, shear strength and coupled hydro-mechanical behaviour of unsaturated soils.

\subsection{Volume change behaviour}

The volume change caused by suction increase (drying) can be predicted by Eqs. 1 and 2 directly. Figure 1 shows the SFG prediction compared with experimental of Mariho et al. (1995). There are three parameters involved in the prediction: the compressibility of saturated soil $\left(\kappa_{v p}\right.$ and $\lambda_{v p}$ ) and the transition suction (air entry value in this case). The figure shows that the SFG model is capable of capturing the volume change during drying.

The volume change caused by stress change can also be predicted by Eq. 1. Figure 2 compares the SFG predictions with the experimental data of Thu et al. (2007). The data were obtained from isotropic compression tests under constant suctions on compacted Kaolin. The predicted results match very well the experimental data, indicating that 


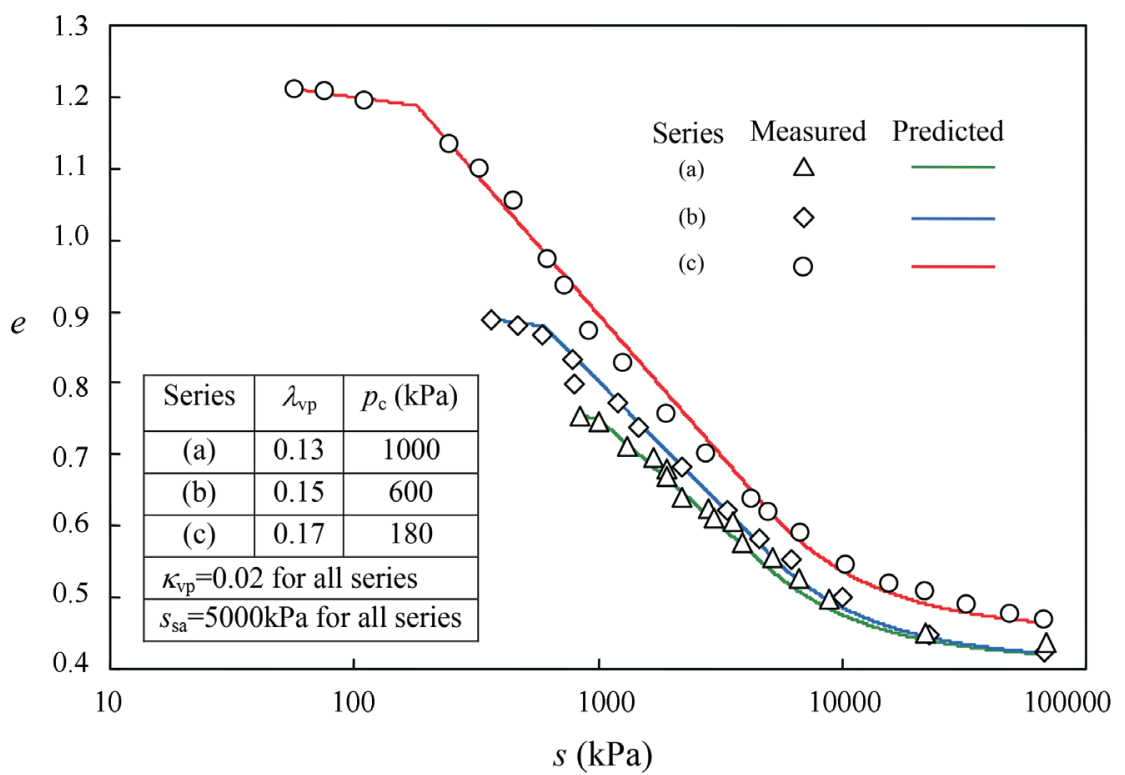

Figure 1 - Volume change during drying for Brown London Clay (data of Marinho, et al., 1995).

Material parameters used in the prediction

\begin{tabular}{|c|c|c|c|c|c|l|}
\hline & $s, \mathrm{kPa}$ & $\lambda_{\mathrm{p}}$ & $\kappa_{\mathrm{vp}}$ & $\bar{p}_{\mathrm{c}}$ & Data & Predictions \\
\hline (a) & 0 & 0.058 & 0.018 & $25 \mathrm{kPa}$ & $\bullet$ & \\
\hline (b) & 50 & 0.058 & 0.018 & $35 \mathrm{kPa}$ & $\square$ & \\
\hline (c) & 100 & 0.058 & 0.018 & $25 \mathrm{kPa}$ & $\Delta$ & \\
\hline (d) & 150 & 0.058 & 0.018 & $35 \mathrm{kPa}$ & $\diamond$ & \\
\hline
\end{tabular}
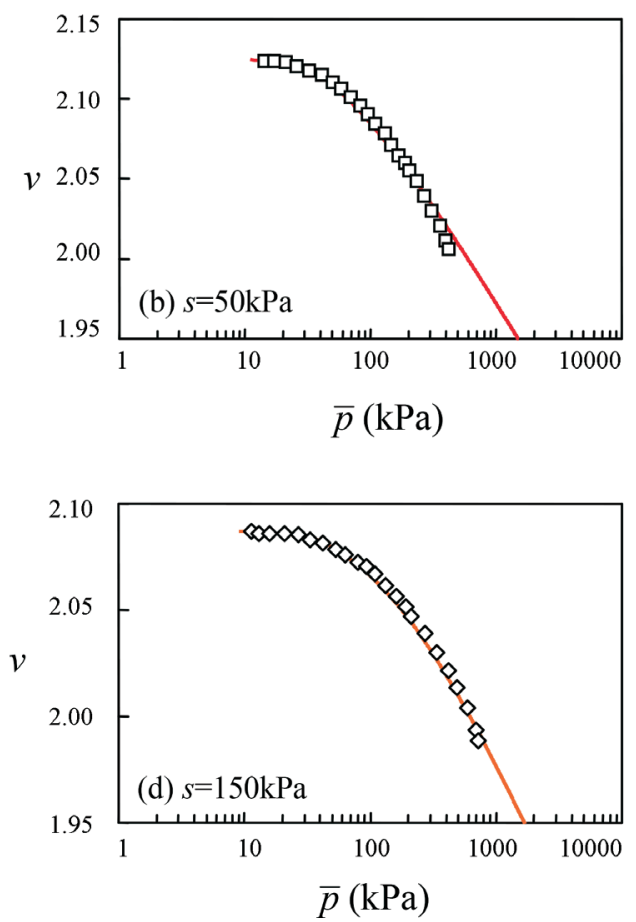
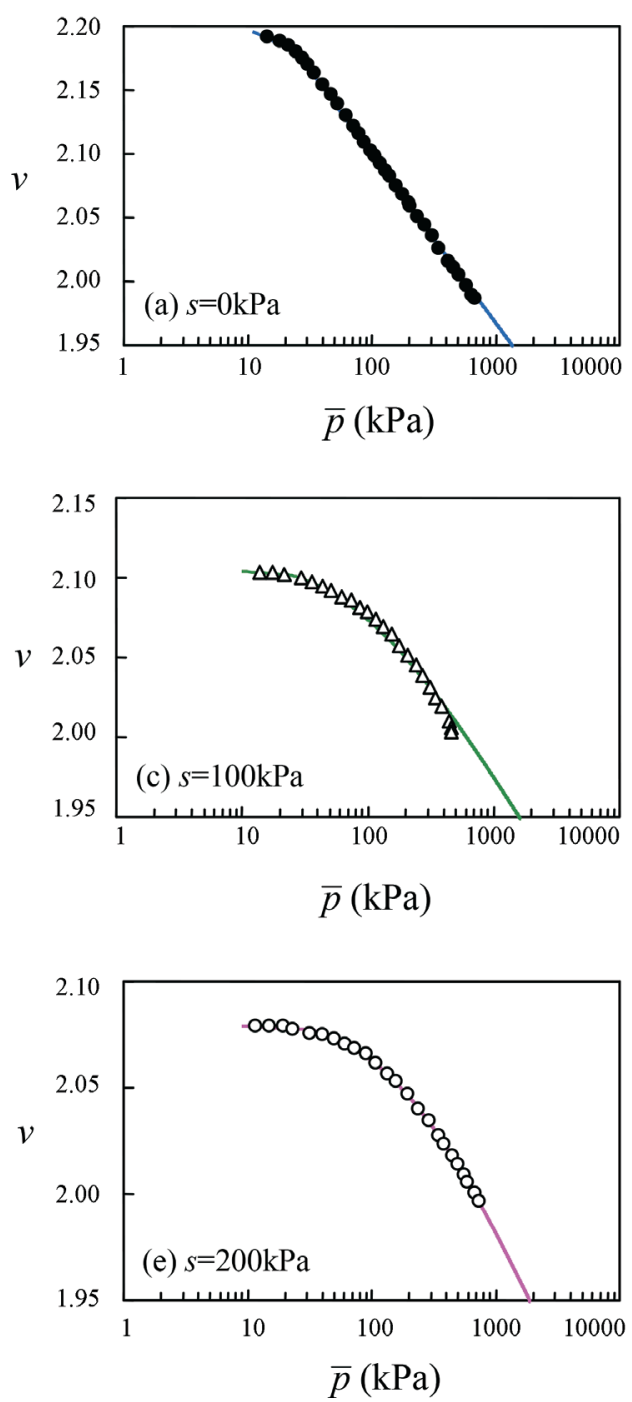

Figure 2 - Predictions of isotropic compression curves for compacted kaolin specimen (data by Thu et al., 2007). 
the SFG model is capable of predicting the volume change caused by stress change.

The volume change during wetting is more complex. It may involve the expansion of the loading-collapse yield surface, or so-called hardening. In this case, a complete constitutive model is needed to predict the volume change. Figure 3 was taken out from Sheng et al. (2008a) and shows the predicted volume collapse during wetting of compacted Pearl clay. The compacted sample (point A in Fig. 3) was first dried to a constant suction (point B) and then compressed to different stress levels (Points D, E, F, G, H, I), followed by wetting under constant net mean stresses full saturation (Points D', E', F', G', H', I'). Figure 3 shows that the predicted collapse volume depends on the stress level where the wetting takes place. The prediction was not compared with experimental data due to the lack of detailed soil parameters. However, the experimental data was shown on the lower left corner, to indicate the qualitative agreement between the prediction and the experimental data.

\subsection{Shear strength}

In the SFG model, the shear strength behaviour is a natural outcome of the volume change equations and does not need any additional definition or material parameters. Nevertheless, the model is capable to predict the shear strength variation with suction to a reasonable degree. Figure 4 shows the predicted shear strength variation with suction, compared with the experimental data by Vanapalli $e t$ al. (1996). The tests and predictions are for direct shear tests. Figure 5 compares the predictions with experimental data from triaxial tests. The results in both figures indicate that the SFG model provides a reasonable prediction of unsaturated soil shear strength.

\subsection{Hydro-mechanical coupling}

The extended SFG model by Sheng \& Zhou (2011) and Zhou et al. (2012a) provides a complete coupling mechanism for the interaction between stress-strain and water retention behaviour. The effects of suction and degree of saturation on stress-strain and strength behaviour have already been demonstrated above. The effects of stress on water retention behaviour are demonstrated below.

Considering the soil water characteristic curves (or SWCC) under constant stresses, the effect of stress is primarily reflected through its effect on the initial soil density

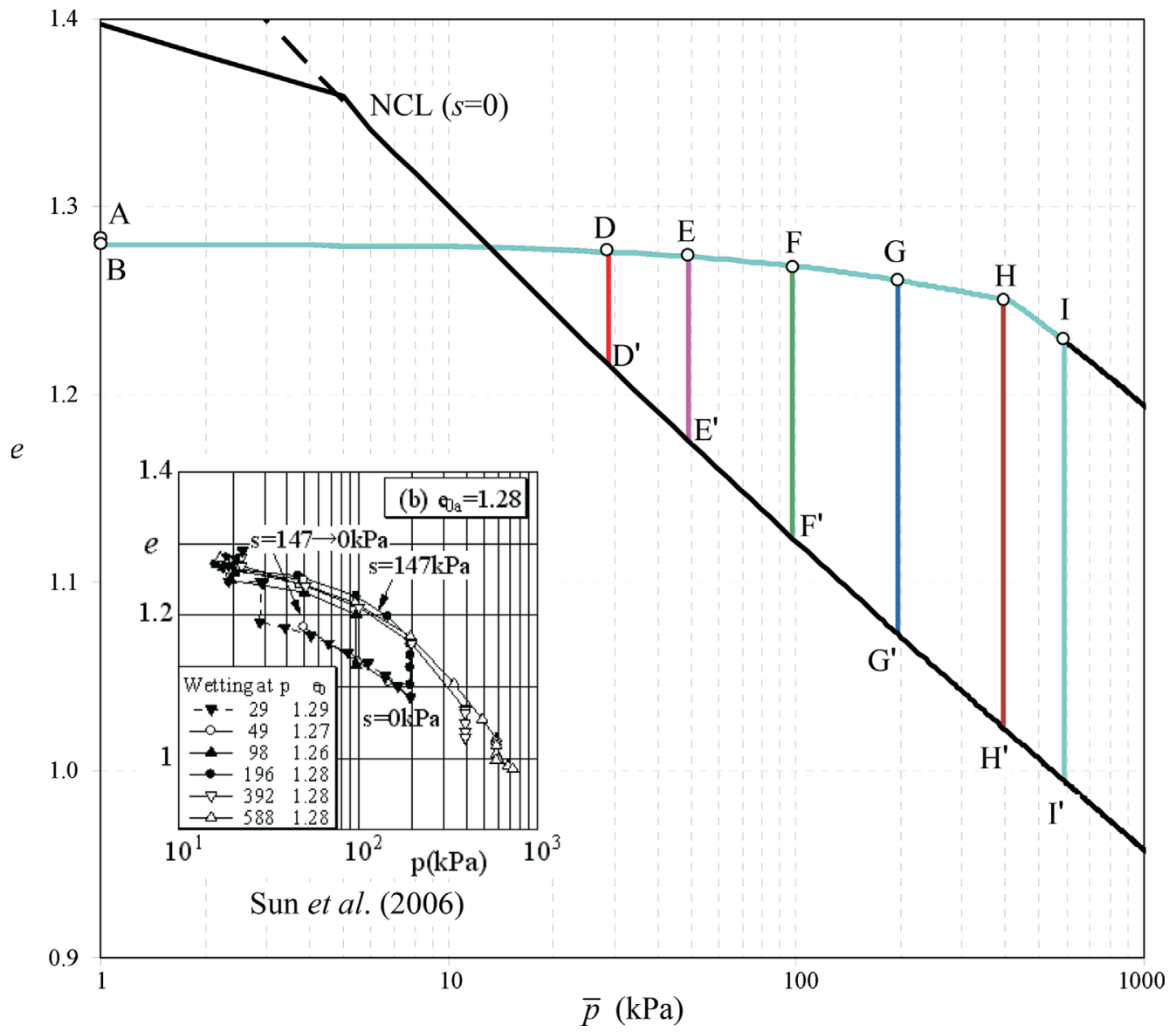

Figure 3 - Predicted volume collapse during the final wetting paths (experimental results of Sun et al. (2007) shown on the lower left corner). 


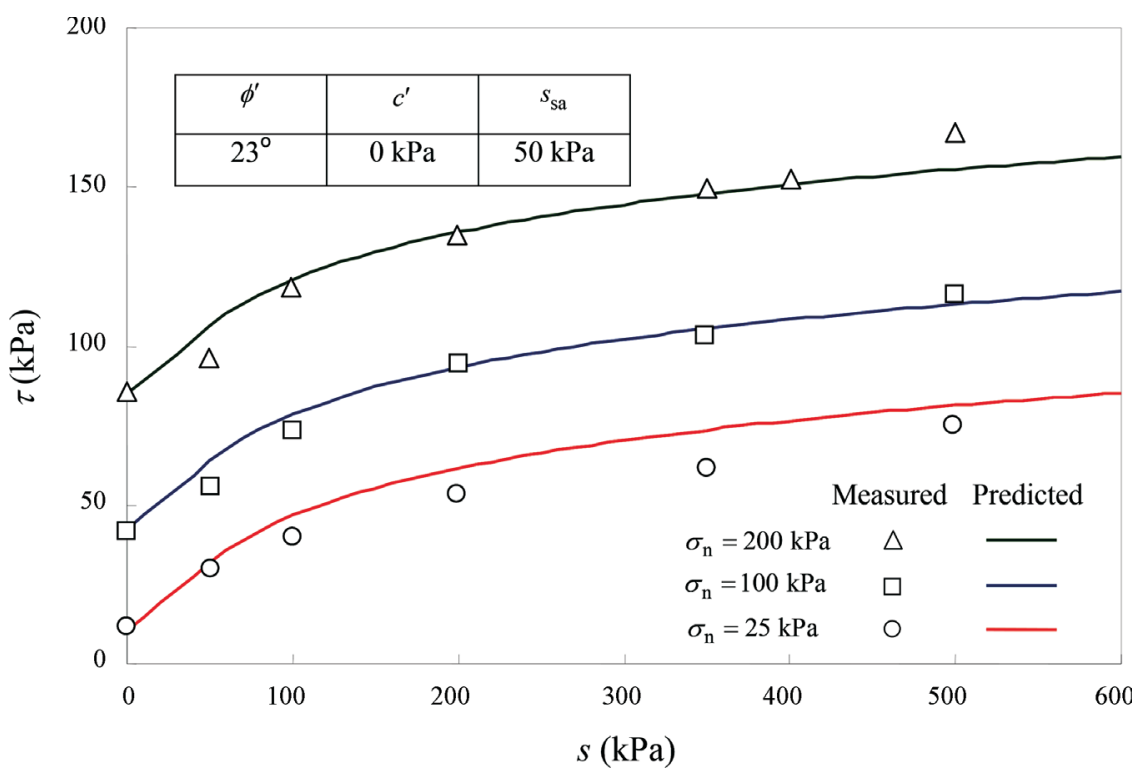

Figure 4 - Shear strength vs. suction during direct shear tests (data by Vanapalli et al., 1996).

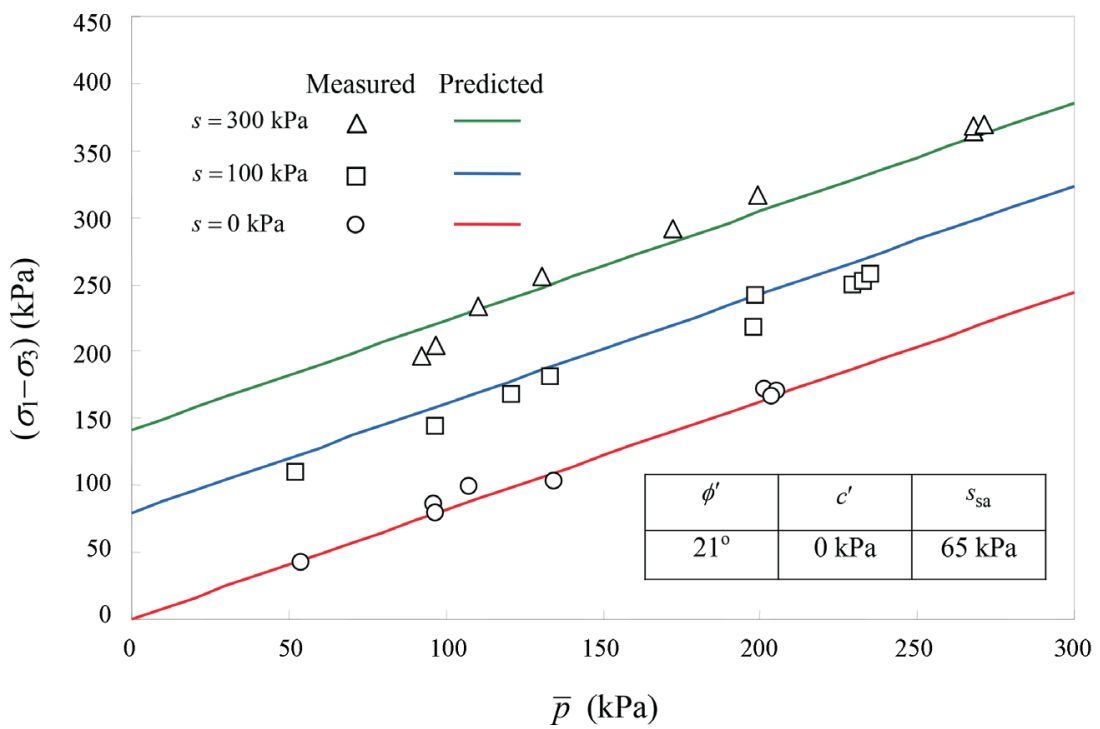

Figure 5 - Shear strength vs. suction during triaxial compression tests (data by Wheeler \& Sivakumar, 2000).

or initial soil void ratio. Figure 6 shows the predicted and measured SWCCs for soils compacted to different initial void ratios. The predicted SWCCs are shown as solid curves. The fitting parameter $m$ in Eq. 8 is set to 0.03 . The SWCC for initial ratio of 0.517 was obtained using van Genuchten equation with fitting parameters given in Sheng \& Zhou (2011). The predicted SWCCs compares very well with the measured data, indicating that the proposed model can capture the shift of SWCC with initial void ratio.

Figure 7 further demonstrates the capability of the SFG model in predicting the effects of initial void ratio on the water retention behaviour. The soil tested was a silty sand from a Saskatchewan Department of Highway borrow pit (Huang et al., 1998). The air-dried silty sand was mixed with distilled water to prepare slurry specimens for the ex-

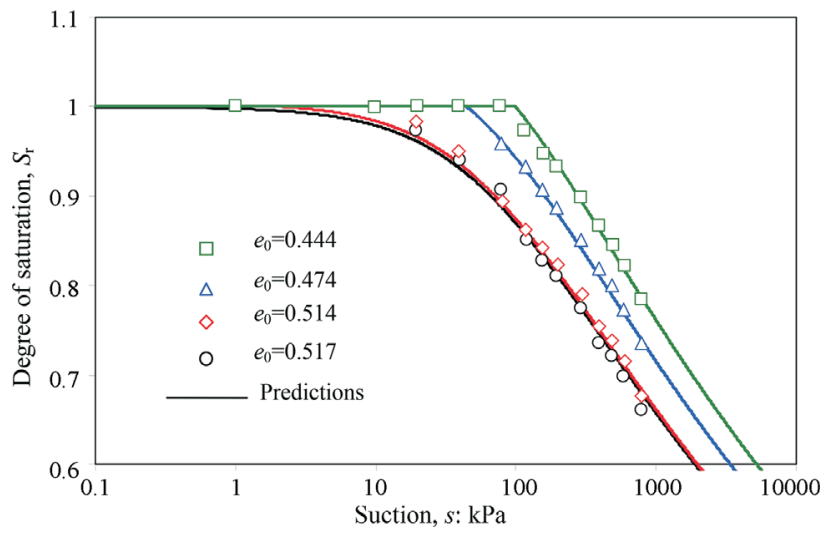

Figure 6 - Measured and predicted SWCCs (data after Vanapalli et al., 1999). 
(a) 1.2

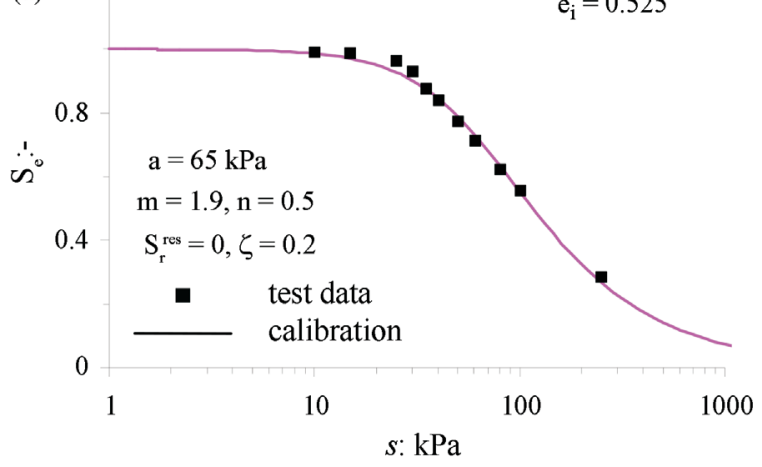

(c) 1.2

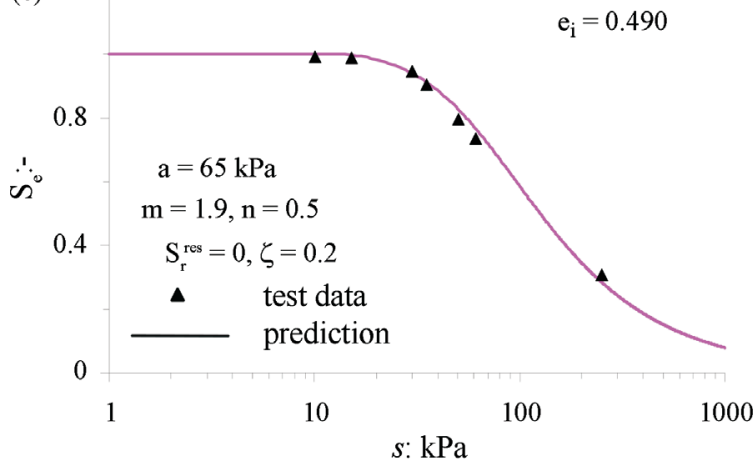

(e) 1.2

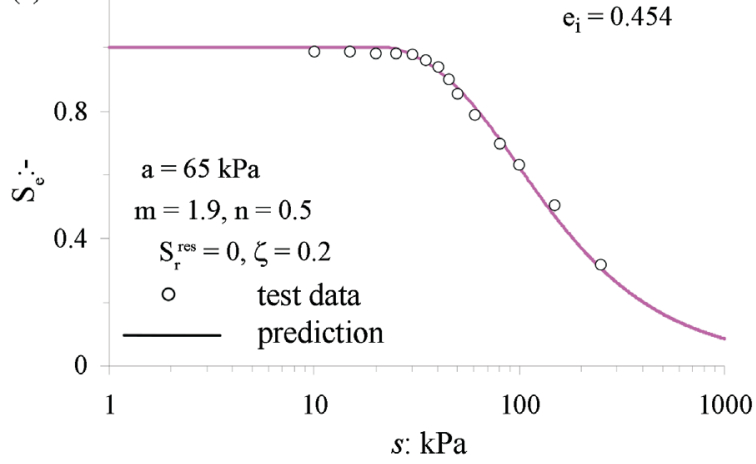

(b) 1.2

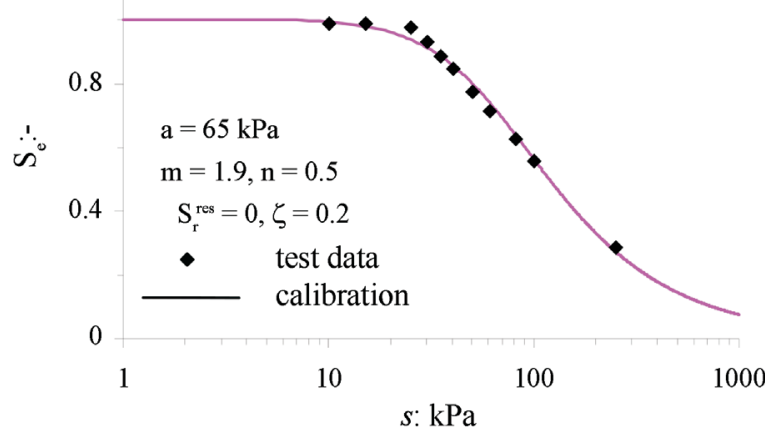

(d) 1.2

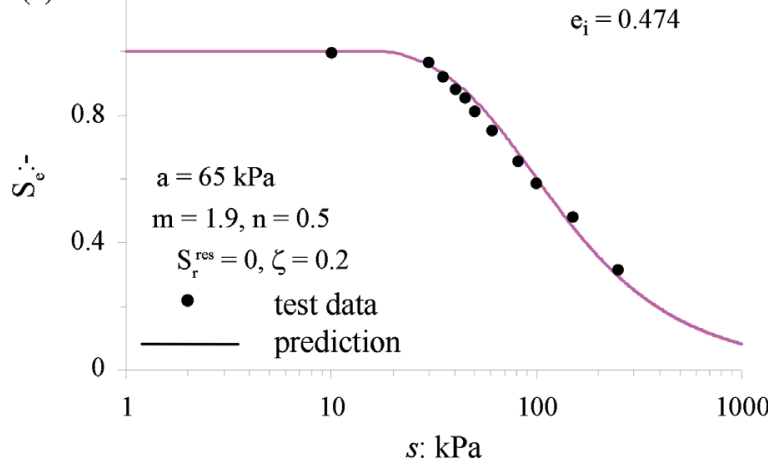

(f) 1.2

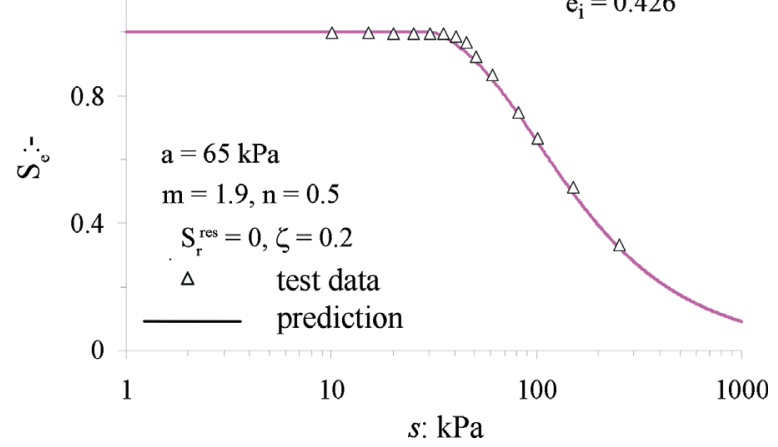

Figure 7 - Measured and predicted SWCCs for a silty sand with different initial void ratios (data after Huang et al., 1998).

perimental program. Six initially slurry specimens were one-dimensionally preconsolidated under different pressures to obtain different initial void ratios for the water retention tests. The test results are replotted in the $S_{\mathrm{e}}-s$ plane together with the predictions of Eq. 8, as shown in Fig. 7. The water retention test data for an initial void ratio 0.525 were used to calibrate the reference van Genuchten SWCC equation (Zhou et al., 2012a). The fitting parameter $m$ in Eq. 8 was set to 0.2. As shown in Figure 7, the predicted SWCCs agree very well with the experimental SWCCs. The shifting of the SWCC due to changes in the initial density is well portrayed by the SFG model.

\section{Limitations of the SFG Model}

\subsection{Loading collapse surface for coupled hydro-mecha- nical formulation}

One common limitation of models defined in the stress - suction space is related to the coupling between water retention and stress-strain behaviour. The SFG model is no exception for this shortcoming.

It is commonly accepted that the water retention curve of a soil depends on the stress level and the initial state (such as initial density) of the soil. Under the same stress level, the main drying curve shifts towards higher suction region with decreasing initial void ratio (Fig. 6). For the 
same soil, the main drying curve shifts towards higher suction region when the stress level applied to the soil increases. That means the air entry suction is not constant for the same soil, but depends on the initial void ratio of the soil and the stress level applied to the soil. On the other hand, the loading-collapse yield curve experiences a curvature change around the transition suction such as the air entry value, which means the shape of the loading-collapse curve depends on the air entry value. Because of the dependency of the air entry value on the stress level and initial condition of the soil, the shape of the load-collapse curve would then depend on the stress level and initial condition of the soil, which is not acceptable for the definition of a yield surface. The yield surface must be independent of the stress states it encloses.

This limitation is common to all models defined in the stress - suction space, irrespective of the stress variables used (Fig. 8). It should also be noted that the transition suction appears implicitly in all models, even though some models set it to zero (such as in BBM of Alonso et al., 1990). As mentioned in Sheng (2011), different degrees of saturation are only states of the soil and the transition from saturated to unsaturated states (for example, a change of pore water pressure from $10 \mathrm{kPa}$ to $-10 \mathrm{kPa}$ ) should always be allowed in a model. Therefore it is not possible to get rid of the transition suction. The conclusion is then that the

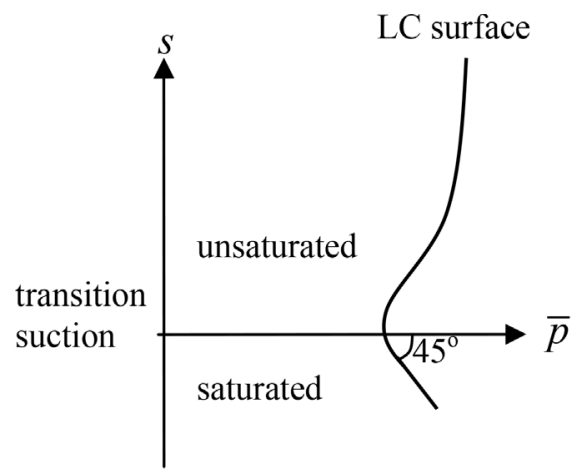

(a) net stress - suction space

Figure 8 - Loading-collapse yield surface in suction-stress space.

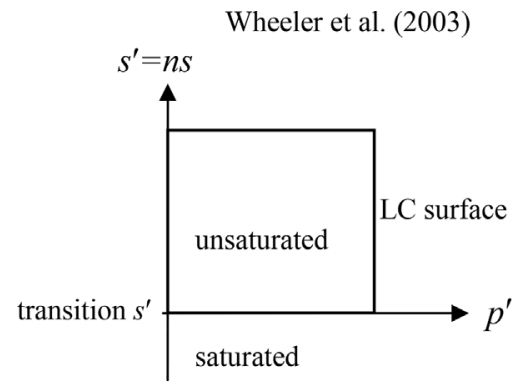

(a) effective stress - modified suction space loading-collapse yield surface becomes meaningless in the stress - suction space if the water retention behaviour is considered to depend on stress level or initial condition of the soil.

A possible solution to the above shortcoming is to define the loading-collapse curve in terms of a variable alternative of suction. In the model by Wheeler et al. (2003), the loading-collapse curve is defined in the space of effective stress vs. modified suction, where it does not have an inflection point (Fig. 9a). Such a definition essentially implies that the water retention curve when plotted in terms of modified suction and degree of saturation is independent of stress levels or initial density of the soil. This implication remains to be further validated experimentally. In the model of Zhou et al. (2012b, 2012c), the loading-collapse curve is defined in the space of effective stress $v s$. degree of saturation and there is no inflection point along the loading-collapse curve in this space (Figure 9b). The loading-collapse curves in these models are then independent of the stress or suction path within the loading-collapse curves and are hence more meaningful.

\subsection{Compression under constant suction}

The current version of the SFG model predicts typical normal compression curves under constant suctions as shown in Fig. 10. In reality, compression under constant

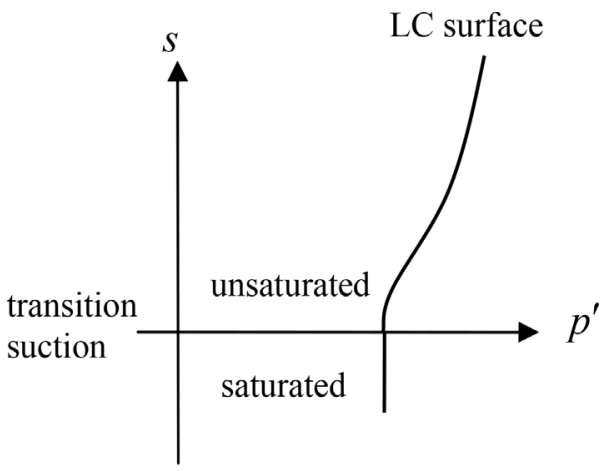

(b) effective stress - suction space 


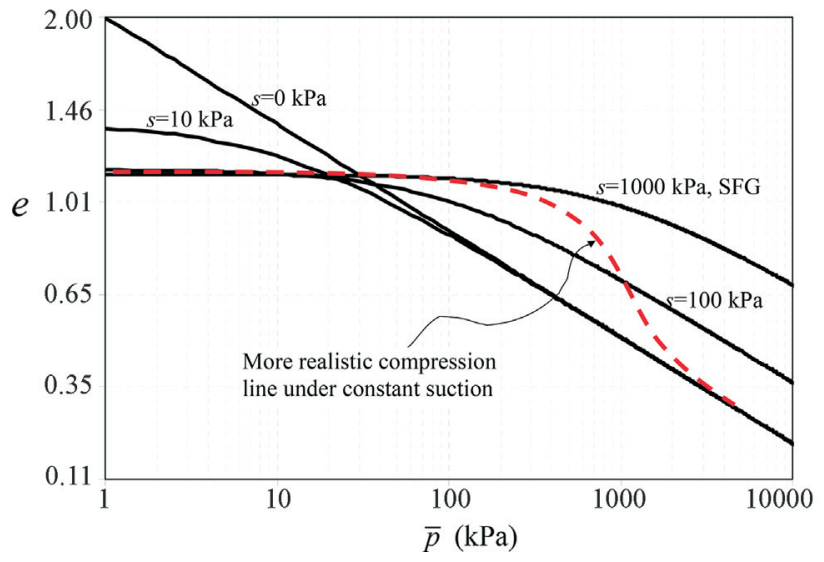

Figure 10 - SFG normal compression lines at different suctions $\left(N=3, \lambda_{v p}=0.1, s_{\mathrm{sa}}=10 \mathrm{kPa}\right)$.

suction, especially under a low suction, results in increase of degree of saturation. In other words, it is possible to compress an unsaturated soil under constant suction to full saturation. A more realistic compression curve for constant suction should then converge to the saturated normal compression line, as shown by the dashed curve in Fig. 10.

This limitation can in theory be overcome by using a compressibility parameter $\left(\lambda_{v p}\right)$ that increases with increasing suction. However, such a modification would significantly complicate the integration of incremental equations in the SFG model. In addition, it would not provide a smooth convergence to full saturation. A better solution is, as suggested in Zhou et al. (2012b), that the compressibility parameter is assumed to decrease with decreasing degree of saturation. With such an assumption, compression under constant suction would result in increase in the degree of saturation and hence decrease in the soil compressibility. However, as pointed out in Sheng (2011), it should be avoided to have both suction and degree of saturation in the volume change equation, due to the hysteretic relationship of the two variables. Introducing the degree of saturation into the volume change equation would require the elimination of suction from it. The effects of suction have then to be considered through the stress variable - effective stress. This is the fundamental reason why Zhou et al. establish their model in the space of effective stress vs. the degree of saturation.

Other limitations of the SFG model include the nonconservative behaviour of the elastic zone, the difficulty of using a more advanced function $f(s)$ in Eq. 1, and the non-convexity of the elastic zone, as discussed in Sheng (2011). However, these limitations are not unique to the SFG model and the measures for dealing with them have been discussed in detail in Sheng (2011).

\section{Summary}

The SFG model provides a consistent and unified framework for describing volume change, yield stress, shear strength and coupled hydro-mechanical behaviour of unsaturated soils. It is established in the space of the independent stress variables, namely net stress and matric suction. It provides a smooth and natural transition between saturated and unsaturated states. The model has been further extended to cover soil behaviour such as hydro-mechanical interaction, hysteretic water retention, and density dependency. However, this model also has a number of limitations that require further improvements. One of the most significant limitations is related to the definition of the loading-collapse yield surface when the coupled hydromechanical behaviour is considered. This paper has presented a summary of the latest developments of the SFG model and the areas where the model falls short of real soil behaviour.

\section{References}

Alonso, E.E.; Gens, A. \& Josa, A. (1990). A constitutive model for partially saturated soils. Geotechnique, 40(3):405-430.

Fredlund, D.G.; Morgenstern, N.R. \& Widger, A. (1978). Shear strength of unsaturated soils. Canadian Geotechnical Journal, 15(3):313-321.

Huang, S.; Barbour, S.L. \& Fredlund, D.G. (1998). Development and verification of a coefficient of permeability function for a deformable unsaturated soil. Canadian Geotechnical Journal, 35(3):411-425.

Marinho, F.A.M.; Chandler, R.J.; \& Crilly, M.S. (1995). Stiffness measurements on an unsaturated high plasticity clay using bender elements. In Proceedings of the 1st International Conference on Unsaturated Soils, September 1995. Paris. A.A. Balkema, Rotterdam, v. 2, pp. 535-539.

Sheng, D. (2011). Review of fundamental principles in modelling unsaturated soil behaviour. Computers and Geotechnics, 38:757-776.

Sheng, D.; Fredlund, D.G. \& Gens, A. (2008a). A new modelling approach for unsaturated soils using independent stress variables. Canadian Geotechnical Journal, 45(5):511-534.

Sheng, D.; Gens, A.; Fredlund, D.G. \& Sloan, S.W. (2008b). Unsaturated soils: From constitutive modelling to numerical algorithms. Computers \& Geotechnics, 35:810-824.

Sheng, D. \& Zhou, A.N. (2011). Coupling hydraulic with mechanical models for unsaturated soils, Canadian Geotechnical Journal, 48(5):826-840.

Sun, D.A.; Sheng, D. \& Xu, X.F. (2007). Collapse behaviour of unsaturated compacted soil. Canadian Geotechnical Journal, 44(6):673-686.

Thu, T.M.; Rahardjo, H. \& Leong, E.C. (2007). Soil water characteristic curve and consolidation behaviour of a compacted silt. Canadian Geotechnical Journal, 44(3):266-275. 
Vanapalli, S.K.; Fredlund, D.G.; Pufahl, D.E. \& Clifton, A.W. (1996). Model for the prediction of shear strength with respect to soil suction. Canadian Geotechnical Journal, 33(3):379-392.

Vanapalli, S.K.; Fredlund, D.G. \& Pufahl, D.E. (1999). The influence of soil structure and stress history on soilwater characteristics of a compacted till. Géotechnique, 49(2):143-159.

Wheeler, S.J.; Sharma, R.S. \& Buisson, M.S.R. (2003). Coupling of hydraulic hysteresis and stress-strain behaviour in unsaturated soils. Géotechnique, 53(1):4154.

Wheeler, S.J. and Sivakumar, V. (2000). Influence of compaction procedure on the mechanical behaviour of an unsaturated compacted clay. Part 2: Shearing and constitutive modelling. Géotechnique, 50(4):369-376.
Zhou, A.N. \& Sheng, D. (2009). Yield stress, volume change and shear strength behaviour of unsaturated soils: Validation of the SFG model. Canadian Geotechnical Journal, 46(8):1034-1045.

Zhou, A.N.; Sheng, D. \& Carter, J.P. (2012a). Modelling the effect of initial density on soil water characteristic curves, Géotechnique, 62(8):669-680.

Zhou, A.N.; Sheng, D.; Sloan, S.W. \& Gens, A. (2012b). Interpretation of unsaturated soil behaviour in the stress - saturation space, I: Volume change and water retention behaviour. Computers and Geotechnics, 43:178187.

Zhou, A.N.; Sheng, D.; Sloan, S.W. \& Gens, A. (2012c). Interpretation of unsaturated soil behaviour in the stress - saturation space, II: Constitutive relationships and validations. Computers and Geotechnics, 43:111-123. 